\title{
OCUPACIÓN Y LITERATURA, UN ANÁLISIS DESDE LA DIALÉCTICA MATERIALISTA
}

\section{Occupation and dialectics, an analysis from Materialistic Dialectic}

\author{
Rolando Ramírez P. ${ }^{\mathrm{i}}$ Marjorie Schliebener T. ${ }^{\mathrm{ii}}$

\section{RESUMEN}

La necesidad de generar conocimiento en torno al estudio de la ocupación en un contexto Latinoamericano lleva a replantear los enfoques y métodos actualmente utilizados para estos fines. Dentro de esto, consideramos que la literatura latinoamericana es una fuente de realidades ocupacionales, ya que se ha caracterizado a nivel mundial por el fuerte contenido histórico en sus narraciones, brindando cotidianeidades que enriquecen el análisis de las ocupaciones en un contexto real, con elementos propios de nuestra historia y nuestra cultura. Para abordar la complejidad de esta búsqueda, se propone la dialéctica materialista como fuente necesaria de desarrollo, que nos permite abordar no solo la complejidad de los elementos en juego, sino además comprender los procesos desde el movimiento y desde su historicidad. Es así que en el presente trabajo, y como ejercicio inicial en esta compleja temática, realizamos el análisis de una ocupación extraída de un libro de literatura chilena del siglo XX, nos referimos a Hijos del Salitre, de Volidia Telteiboim. En este ejercicio analítico se observan diversos aspectos de la ocupación a través de categorías dialécticas, que nos permiten, en primera instancia explorar diversos ámbitos del fenómeno ocupacional que actualmente no habían sido considerados. Finalmente, y como proceso necesario para el aprendizaje y el desarrollo de conocimiento, es que realzamos autocríticas y auto observaciones del mismo ejercicio de análisis realizado.

\section{ABSTRACT}

The need to generate knowledge related to the study of occupation within a Latin-American context leads to reformulate the focuses and methods currently used for these ends.

Furthermore, we consider that Latin American literature is a source of occupational realities, since it has been characterized, in a Worldwide level, for its Strong historical content in its narrations, providing quotidian events which enrich the analysis of occupations in a real context, with elements of our own culture and history.

\footnotetext{
${ }^{\text {i }}$ Licenciado en Ciencia de la Ocupación y Terapeuta Ocupacional, Gendarmeria de Chile, C.D.T. Santiago Sur, 8-2906056, rolando.ramirez.p@gmail.com

${ }^{\text {ii } L i c e n c i a d a ~ e n ~ C i e n c i a ~ d e ~ l a ~ O c u p a c i o ́ n ~ y ~ T e r a p e u t a ~ O c u p a c i o n a l, ~ C O S A M ~ Q u i n t a ~ N o r m a l, ~ 8-4245383, ~}$ mlschliebener@gmail.com
} 
To approach the complexity of this search, materialistic dialectics is proposed, as a necessary source of development, which allow us to approach not only the complexity of elements in play, but also to understand the processes from the movement and from its historicity.

Thus, in the present paper, and as an initial exercise in this complex theme, we make the analysis of an occupation extracted from a book of 20th Century Chilean literature , we refer to "Hijos del Salitre" (Children of nitrate), from Volodia Telteiboim. In this exercise, diverse aspects of occupation are observed, through dialectic categories, which allow us, at the first instance, to explore different areas of occupational phenomenon which nowadays were not considered

Finally, and as a necessary proccess to learning and development of knowledge, we make self-criticisms and self-observations of the same exercise analisys excercise carried out.

Palabras claves: Ocupación, dialéctica materialista, categorías dialécticas, literatura chilena.

Key words: Occupation, dialectical materialist, dialectical categories, chilean literature

\section{INTRODUCCIÓN}

\section{Ocupación y Literatura Latinoamericana...Una mirada desde la dialéctica.}

La ocupación, ha estado siendo recientemente estudiada y sistematizada por la necesidad de comprenderla no sólo desde el punto de vista terapéutico, sino además, desde una perspectiva más universal. Es así que en 1989 es fundada en Estados Unidos la Ciencia de la Ocupación, enfoque teórico que pretende dar bases y fundamentos científicos a la Ocupación, como manifestación del que hacer humano en todas sus esferas. Las complicaciones para su abordaje no han estado ausentes, y se ha llegado al cuestionamiento de que la Ciencia de la Ocupación no es aplicable en todos los lugares de igual manera, ya que los sujetos que realizan éstas ocupaciones viven en contextos similares y a la vez muy diferentes. Se ha venido discutiendo la propuesta de que cada región del globo debería brindar sus aportaciones teóricas a la construcción de este nuevo paradigma. Es por eso que surge la necesidad de abordar nuevos enfoques que nos permitan estudiar y comprender a la ocupación en su complejidad y en su desarrollo, más allá de una simple descripción parcial, segmentada y reducida al sujeto que la ejecuta.

El ser humano se define por su naturaleza social, y es bajo esta condición donde se aprenden, ejecutan y desarrollan sus ocupaciones. "Plantear que el individuo es social no significa (...) que lo que hagan los individuos no importa. La historia se desarrolla y ocurre a través de las acciones de individuos concretos, (...) lo que hace el individuo no se puede explicar a partir de una teoría individualista porque el individuo incorpora características que son sociales, propiedades colectivas. Esto significa que no se pueden definir intereses 
individuales con independencia de las propiedades del grupo, o la clase social, a la que pertenece el individuo (Howard y King, 2000) ${ }^{1}$.

Así, el humano se concibe a si mismo en relación a los demás e influye tanto a él como a su entorno mediante sus actos. La transformación de este entorno va modificando las condiciones contextuales originales las que a su vez vuelven a influir, bajo nuevas condiciones, a quién las modificó en principio, transformándolo a su vez. Es por esto que debemos entender a la ocupación, en constante transformación, en constante movimiento, en un constante devenir. La ocupación de ayer, aun teniendo la misma forma aparente que la hoy, son distintas, influyen de distinta manera, se encuentran en otro tiempo, en otro espacio, aunque a simple vista no se aprecie.

La dialéctica, entendida como "la ciencia sobre las leyes generales del movimiento y desarrollo de la naturaleza, sobre la concatenación universal de todos los fenómenos existentes en el universo" ${ }^{2}$, nos permite abordar el estudio de la ocupación desde su movimiento, desde su dinamismo y por ende con sus concatenaciones con los demás fenómenos, tanto sociales como naturales.

Al entender a la ocupación desde el movimiento, desde su transformación, la debemos comprender además desde su materialidad, es decir la ocupación entendida no como la simple idea surgida de la cabeza de quién la ejecuta. Ésta surge desde la realidad (factor objetivo), es aprendida en la sociedad por sus miembros (factor subjetivo) y devuelta a ella como acción, como actividad, como ocupación (síntesis de ambos factores). No es casual que la ocupación de cocinar, sea tan distinta en el siglo I, que en el siglo XV o en el siglo XI. Responde a distintos contextos, a distintas necesidades, surgidas de la misma sociedad que a través de la ocupación va desarrollando condiciones distintas, necesidades distintas que van a su vez transformando a la misma ocupación. O sea, la ocupación ha respondido a una necesidad material, donde" en última instancia, el factor subjetivo depende de las condiciones objetivas de vida. Éstas determinan el contenido y las funciones de dicho factor, al tiempo que desempeñan un papel activo en la transformación de la realidad"3.

Bajo estas concepciones, debe necesariamente entenderse además, en su desarrollo histórico, con sus características históricas, necesidades y condiciones sociales que la han llevado al punto en que estamos hoy.

Considerando la determinación de lo histórico en el estudio de la ocupación, es que aparece como necesario desarrollar y caracterizarla desde nuestro contexto más próximo, que es a nivel general, la gran Latinoamérica, y a nivel particular, Chile, utilizando como fuente, en el presente estudio, la destacada y abundante literatura de nuestra región.

La literatura latinoamericana se ha caracterizado a nivel mundial por el fuerte contenido histórico en sus narraciones, tales como: Las Venas Abiertas de Latinoamérica, de Eduardo Galeano; Subterra de Baldomero Lillo; Tengo Miedo Torero, de Pedro Lemebel, entre otros. 
Es así que nos encontramos a través de diversas obras con la problemática indígena, con las cotidianeidades rurales, con los largos periodos de dictaduras y rebeliones, el tema de la esclavitud y el trabajo de los obreros de la caña de azúcar, de las minas, del café, las mitologías, etc. Así, el lector ya no es un factor ajeno, un marginal de la literatura: no sólo se siente tema, y por tanto se reconoce en la obra de arte, sino que además se siente cómplice. Si la realidad social, penetra de algún modo en el individuo, también el personaje se convierte en social.

Es de esta forma que podemos decir que la literatura latinoamericana construye identidad cultural y también es fuente histórica de procesos, de realidad social, pero también de cotidianeidades.

Enrique Pichón Riviére define a lo cotidiano como la expresión inmediata del orden social: "Las formas concretas que reviste la vida de los hombres están directamente relacionadas con las modalidades en que la existencia material se produce y se reproduce. El objeto, los medios y las formas de producción, así como la inserción de los sujetos en ese proceso productivo, la distribución de lo producido y la relación que guarda lo que se produce y su distribución con las necesidades de los hombres que constituyen una organización social, determinan sus formas de vida, su cotidianeidad." 4

Es así que el contexto socio-histórico interactúa y modifica la cotidianeidad de las individualidades y al revés, transformándose constantemente.

De esta forma, y dentro de la riqueza de la descripción de cotidianeidades marcadas en un contexto que la literatura Latinoamérica nos entrega, es que se convierte en una fuente nutrida de información para analizar las ocupaciones que en estas se describen. En cada libro hay ocupaciones, porque en cada libro se desarrollan vivencias de personas, tanto a nivel de los hechos que experimentan, tanto así como de qué forma lo experimentan, lo objetivo y subjetivo de las relaciones, de las formas en que las ocupaciones se realizan, el contenido que detrás de estas formas se guarda, etc.

En cualquier tipo de conocimiento, ya sea cotidiano, científico o artístico, el sujeto y el objeto están en perpetua interacción, ésta por definición es una interacción dialéctica, donde sujeto y objeto se encuentran como opuestos pero son parte de un mismo todo.

Es de esta forma que para considerar la expresión artística, y más específicamente la literaria como un hecho, y no como un supuesto metafísico, debemos observarla y comprenderla como una interacción entre diversos sujetos y objetos inseparables que nos dan a conocer relaciones que suceden, que poseen una historicidad, que son sociales y producen socialización. 
La literatura y los textos literarios parten de contextos reales, de sujetos o prototipos de sujetos reales que se van transformando estéticamente a través del lenguaje o forma de discurso literario creando su propia particularidad.

Antes de que el texto literario se eleve a una abstracción escrita en un libro, la construcción de ésta comienza por la experiencia. Es así que podemos decir que la literatura es práctica.

En este caso se analiza una ocupación de una obra literaria chilena, donde se problematiza diferentes cotidianeidades ocupacionales, desde y hacia el contexto económico-políticocultural donde las historias se desarrollan.

Finalmente se desarrollará un planteamiento crítico de nuestra propuesta, describiendo los límites que se presentaron, las contradicciones propias de la metodología utilizada y las posibles propuestas de superación de esta incipiente propuesta, respondiendo de esta manera a la crítica no sólo como herramienta externa sino como elemento que se desarrolla en el mismo proceso de elaboración.

En consecuencia, el estudio de la ocupación debiera necesariamente abordarse considerando todos los aspectos antes mencionados, para de esta manera poder sintetizar los elementos dispersos y aislados con que actualmente se ha pretendido desarrollar. Todos estos elementos son propios de un análisis dialéctico materialista, el cual partiendo de la realidad material de los fenómenos y objetos, los estudia en su movimiento, en su contradicción, en su totalidad y en su superación crítica. A diferencia de los enfoques científicos tradicionales, los elementos necesariamente parcelados para su estudio, son nuevamente unidos, sintetizados para una comprensión global del fenómeno, para con esto evitar que "los árboles no nos dejen ver el bosque”.

\section{METODO}

Esta investigación corresponde a un estudio exploratorio de tipo analítico, ya que no existe información sobre estudios de ocupaciones en Chile ni en Latinoamérica desde una perspectiva dialéctica materialista, así como también, tampoco existen registros de estudios de ocupaciones extraídas de fuentes históricas tales como textos literarios latinoamericanos.

Este estudio es la etapa inicial de posibles investigaciones futuras dentro de esta área no explorada en la Ciencia de la Ocupación.

\section{Métodos de recolección de datos}

\section{Perspectiva dialéctica materialista}


La dialéctica llevada al lenguage formal academicista podría plantearse como "la ciencia sobre las leyes generales del movimiento y desarrollo de la naturaleza, sobre la concatenación universal de todos los fenómenos existentes en el universo" ${ }^{5}$

En otras palabras es una forma de analizar el universo, que parte del axioma de que todo se encuentra en un estado de constante cambio y flujo. Pero no sólo eso. La dialéctica explica que el cambio y la moción (energía) implican contradicción, y sólo pueden darse a través de contradicciones. El principio fundamental de la dialéctica es que todo está sometido a un proceso constante de cambio, moción y desarrollo. Incluso cuando nos parece que no está pasando nada, en realidad, la materia siempre está cambiando.

El presente estudio basará su fundamento en la dialéctica, pero ¿por qué dialéctica? por la necesidad de abordar nuevas perspectivas que nos permitan estudiar y comprender a la ocupación en su complejidad y en su desarrollo, más allá de una simple descripción parcial, segmentada y reducida al sujeto que la ejecuta.

Y ¿por qué materialista? por la necesidad de comprender además desde su materialidad, es decir la ocupación entendida no como la simple idea surgida de la cabeza de quién la ejecuta, sino desarrollada en una realidad concreta, donde" en última instancia, el factor subjetivo depende de las condiciones objetivas de vida. (TCHaGUin B.A tCHAGUin B.A)

Para efectos de este estudio, se utiliza como fuente de organización y análisis, las categorías de la dialéctica materialista.

Las categorías filosóficas son conceptos que reflejan rasgos y nexos, aspectos y propiedades generales de la realidad.

Las categorías de la dialéctica materialista se diferencian de los conceptos generales de las ciencias. Estos conceptos son aplicables a una esfera determinada del conocimiento. Las categorías materialistas dialécticas impregnan todos los ámbitos del saber. Permiten desentrañar procesos materiales y espirituales altamente complejos.

Estas se presentan como polos contrarios de una misma unidad (contradicción), lo que en su conjunto permiten comprender desde una visión más compleja y dinámica el análisis desarrollado.

La fuente de información utilizada, para establecer la ocupación a analizar, corresponde al libro "Hijos del Salitre", del escritor chileno Volodia Teitelboim ( 1952)

La ocupación elegida corresponde al trabajo minero del salitre, ubicada en la oficina San Lorenzo. 
Los criterios de elección responden a consideraciones relacionadas con la vigencia de esta ocupación, que si bien hoy se realiza de una forma diferente, aún se realizan trabajos en minas, para extraer recursos naturales. Otro criterio fue la importancia histórica y la trascendencia cultural que dejaron las comunidades que vivenciaron el trabajo en las oficinas del salitre, hecho muy característico del norte y de la historia del país. Además, es la ocupación principal de la obra literaria, de la cual se desarrollan una serie de procesos que se originan debido a las condiciones laborales de esta ocupación. Es importante destacar que este libro esta basado en un personaje real que vivió todo el proceso de trabajar en más de una oficina salitrera y de participar en la huelga del salitre, que terminó funestamente con la matanza de la Escuela de Santa María, por lo que está construido con testimonios sobre la base de hechos reales.

\section{RESULTADOS}

La presentación de resultados consiste en el instrumento de descripción primario de la investigación, donde se relaciona la ocupación seleccionada con cada categoría de análisis.

La ocupación señalada será descrita en cada una de las columnas siguientes, correspondientes a las categorías de análisis, respaldadas por citas del texto si fuese necesario. El método de análisis que se realizará en cada cuadro (como forma de análisis

primario) consistirá en el establecimiento de la tesis, antitesis y síntesis de la ocupación por categoría.

Debido a que este tipo de investigación aproximativa, no presenta antecedentes que respalden la relación entre categorías dialécticas y ocupación, es que aparece como necesario incluir las tablas descriptivas como parte del desarrollo del estudio, ya que de esta forma, se mantiene el hilo conductor del trabajo, y el lector conoce y se introduce en esta nueva propuesta. Es así, que sin la lectura correspondiente de cada presentación, el análisis y las conclusiones corren el riesgo de no ser aprehendidas ni comprendidas por quien las conoce, ya que principalmente, se trata de una propuesta nueva en nuestro campo.

\section{Resultados 1}

Libro: Hijos del Salitre

Autor: Volodia Teitelboim

Año: 1952

Ocupación: Trabajo Minero del Salitre, Oficina San Lorenzo, Chile. 


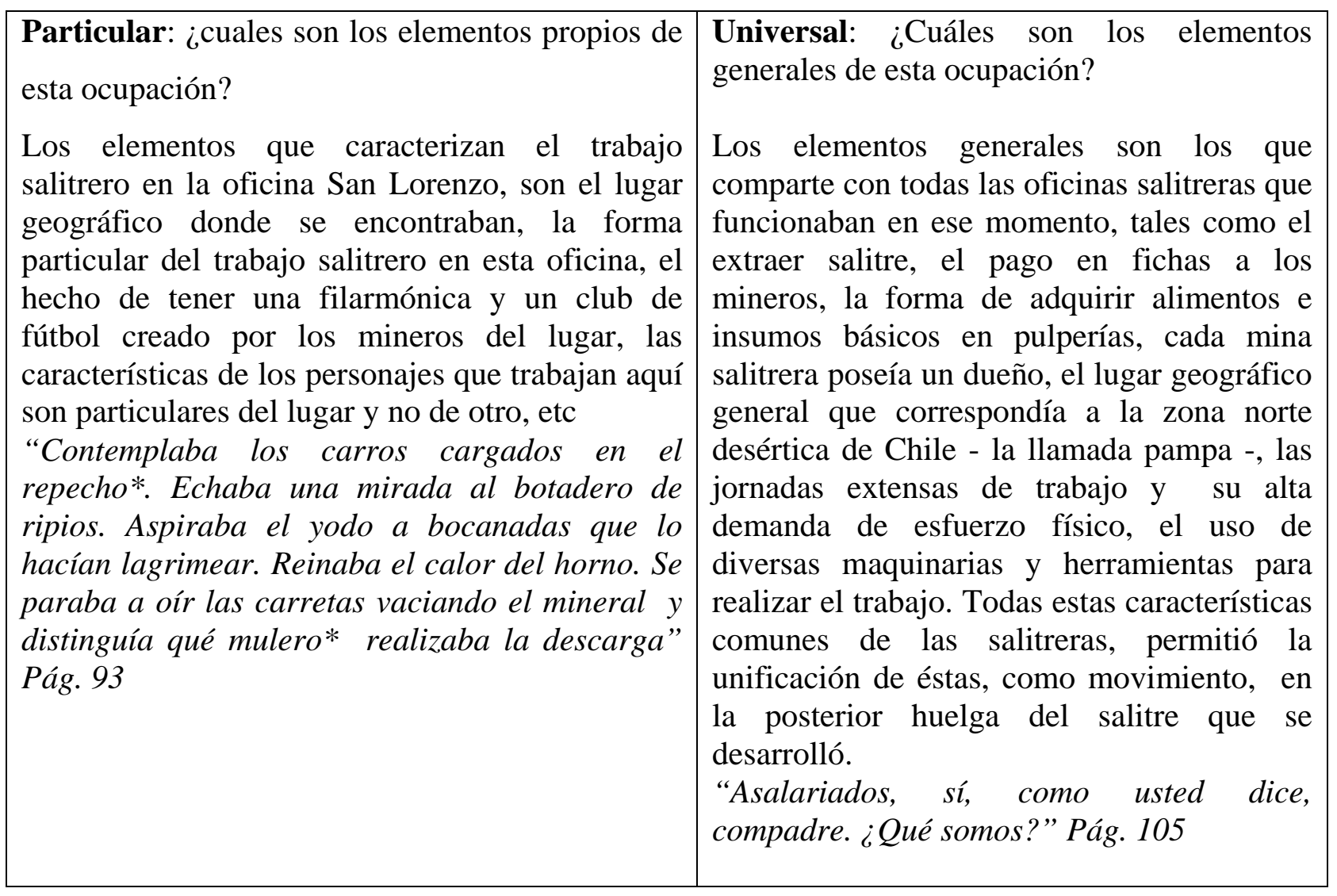




\begin{tabular}{|c|c|}
\hline $\begin{array}{l}\text { Causa: ¿Por qué llego a desempeñarse esta } \\
\text { ocupación? }\end{array}$ & $\begin{array}{l}\text { Efecto: ¿Cuál es el resultado directo de la } \\
\text { causa? }\end{array}$ \\
\hline $\begin{array}{l}\text { En el libro no se describe la causa de por qué se } \\
\text { llegó a desempeñar esta ocupación en esta oficina } \\
\text { salitrera, pero si aparecen elementos que nos } \\
\text { pueden indicar esta información, tales como la } \\
\text { existencia del salitre en ese lugar, la posesión de } \\
\text { recursos económicos de quien es dueño de la } \\
\text { salitrera y la existencia de trabajadores que } \\
\text { desempeñaban este trabajo. La relación entre } \\
\text { estos tres elementos nos indicarían el por qué se } \\
\text { comenzó a desempeñar esta ocupación en este } \\
\text { lugar particular y que se llamó oficina de San } \\
\text { Lorenzo. }\end{array}$ & $\begin{array}{l}\text { El efecto que desencadenó la presencia de } \\
\text { salitre en ese lugar, la existencia de una } \\
\text { persona con recursos para comprar el terreno } \\
\text { y pagarle a trabajadores que desempeñaran la } \\
\text { ocupación; fue que el dueño comenzó a } \\
\text { obtener ganancias de su negocio, que los } \\
\text { trabajadores obtuvieron un salario a cambio } \\
\text { de su trabajo, que brindaba condiciones } \\
\text { laborales particulares (definidas horas de } \\
\text { trabajo, forma de pago, cantidad de salario, } \\
\text { demanda de esfuerzo físico, etc.) y que se } \\
\text { fue extrayendo el salitre de su lugar natural, } \\
\text { transformando la zona geográfica donde éste } \\
\text { se encontraba. } \\
\text { “Descubrió que las manos y los pies } \\
\text { asumían una personalidad expresiva y dura } \\
\text { y que los zapatos polvorientos eran como } \\
\text { parte del hombre. Los gringos sólo querían } \\
\text { sus manos. ¿No decían ellos que los obreros } \\
\text { no valen por su cabeza, sino por las manos y } \\
\text { un poco, además, por las piernas?” Pág. } \\
100\end{array}$ \\
\hline
\end{tabular}


Contenido: ¿Cuáles son los elementos que conforman el proceso de la ocupación?

El contenido se refiere a los elementos presentes en el trabajo minero del salitre y que lo caracterizan como proceso. Estos elementos no salen completamente descritos en el libro, por lo que no podemos expresarlo textualmente es este espacio, pero corresponderían a las siguientes actividades:

Actividades que involucran la extracción del salitre, actividades que involucran el procesamiento del salitre para la venta, venta del salitre, obtención de ganancias.

Los elementos mencionados cubren todo el proceso que componen esta ocupación y que al mismo tiempo les da el sentido o propósito.
Forma: ¿De qué modo se manifiestan los elementos del contenido?

Esta categoría se identifica el "cómo" se realizan y organizan las actividades que componen la ocupación, son la manifestación del contenido. Si bien no aparecen todos los elementos descritos, si se narran algunos. Por ejemplo, la forma en como realiza su trabajo el desrripiador*, cargador, el horario de la jornada de trabajo según la tarea, etc. A continuación podemos citar del mismo libro lo siguiente:

"Elías lo supo por su cuerpo: no es mucha la gente que puede aguantar el oficio de Desrripiador*. El cachucho* no es una pila de agua bendita, sino un fondo hirviente, donde aúlla la piedra rebelde que no se disuelve en un líquido a $120^{\circ}$. Por lo tanto, nadie puede entrar allí vestido como un caballero o un santo. Pág. 107

Necesidad: ¿Qué elementos deben existir para que se realice esta ocupación?

Lo necesario para que se lleve a cabo esta ocupación aparece de forma transversal en todo el libro. Es necesario, para que se lleve a cabo esta ocupación: trabajadores; la existencia de medios de producción, tales como las herramientas, maquinarias, la misma mina; la existencia de un dueño que provea los recursos económicos necesarios para que este trabajo se lleve a cabo; y el recurso natural a extraer, que en este caso es salitre.
Casualidad: ¿Qué elementos pueden 0 no existir para que se realice esta ocupación?

Lo casual en esta ocupación, son los elementos que aparecen, pero no son controlados por quienes participan en el proceso, tales como el origen y características de los mineros, el origen del dueño de la oficina, el clima, etc.

"Nada despedía mayor vida que las manos, requemadas manos de labriegos y pampinos, de changos y aymaras, de chilenos, bolivianos y peruanos, roídas por el tiempo y las herramientas" Pág. 100 
Realidad: ¿Cómo se presenta, en el espacio literario, la ocupación?

La realidad corresponde a como se están presentando, a través de la ocupación, las categorías dialécticas en el tiempo literario real. De esta forma, en esta descripción habría que volver a relatar la forma, fenómeno, particularidad, causalidad, etc. del trabajo del salitre en la oficina San Lorenzo.
Posibilidad: ¿De qué otras formas se podría presentar la ocupación?

La posibilidad corresponde a otras formas en que se podría desarrollar el trabajo salitrero partiendo de la realidad que se describe en el libro. Si bien, esta ocupación se podría desarrollar de muchas formas diferentes, en el libro se narra una posibilidad que proponen los mismos mineros, y que se relaciona con cambios en las condiciones de trabajo que ellos tienen, que se basa principalmente en la forma de pago, en la cantidad de salario y en la forma de adquirir alimentos e insumos básicos. Esto se refleja en la siguiente cita:

"A las diez de la mañana en estado de exaltación, ondeando banderas chilenas y bolivianas, infladas como ventosas, condecoradas de remiendos, y carteles pintados con motes - "Cambio a 18 peniques”, “Baja de los precios”, “Alza de los salarios", "rejas para los cachuchos"caminaron por la gran pampa abierta, rumbo a Santa Lucía, la penúltima oficina del cantón, antes de Gloria, ubicada en los quintos infiernos y aún inconclusa.” Pág. 144 


\begin{tabular}{|c|c|}
\hline $\begin{array}{l}\text { Esencia: ¿Cuál es el aspecto principal de los } \\
\text { elementos que conforman el proceso de la } \\
\text { ocupación? }\end{array}$ & $\begin{array}{l}\text { Fenómeno: ¿Cómo se manifiesta el } \\
\text { aspecto principal de los elementos que } \\
\text { conforman el proceso de la ocupación? }\end{array}$ \\
\hline $\begin{array}{l}\text { El proceso o relación que define en si mismo el } \\
\text { trabajo minero del salitre es la que se da entre el } \\
\text { minero y la extracción del salitre, sin este } \\
\text { proceso, la ocupación en cuestión ya no es la } \\
\text { misma, sino otra diferente. Esta relación } \\
\text { corresponde al aspecto principal en el que se basa } \\
\text { todo el desempeño de esta ocupación. }\end{array}$ & $\begin{array}{l}\text { Esta categoría responde a la forma de la } \\
\text { esencia, no la forma de toda la ocupación, } \\
\text { sino específicamente a la manifestación sólo } \\
\text { de la esencia. En el libro no aparece descrito } \\
\text { el momento en que el minero extrae este } \\
\text { mineral, por lo que no podemos aquí } \\
\text { describir el proceso, ya que sólo nos } \\
\text { acogemos a los antecedentes que el libro nos } \\
\text { entrega. Esta situación no significa que no } \\
\text { exista una manifestación de la esencia, sino } \\
\text { que solo en el libro no aparee este fenómeno } \\
\text { narrado, a pesar de que sí se describen otros } \\
\text { procesos que contienen el trabajo minero del } \\
\text { salitre. }\end{array}$ \\
\hline
\end{tabular}

\section{DISCUSION}

\section{Ventajas}

En cuanto a las ventajas que identificamos en la utilización de esta forma de presentación de resultados destacamos lo siguiente:

- La utilización de las categorías que aparecen en esta propuesta y con las cuales se realizó el proceso de descripción de las ocupaciones, nos propone nuevos ámbitos desde los cuales comprender a la ocupación como fenómeno. Si bien, algunas de las categorías que son propuestas en este estudio, han sido propuestas y desarrolladas por cientistas ocupacionales para nutrir el análisis de las ocupaciones, como la forma por ejemplo, las categorías expuestas nos permiten aumentar el espectro de elementos que componen una ocupación y que se expanden desde el "aquí y ahora" del momento en que se desempeña, abarcando también el antes de que la ocupación se comience a desarrollar, aportando en la identificación de las necesidades de donde surge ésta o la causa directa que genera el fenómeno, pero no sólo aporta en un análisis retrospectivo de los movimientos y procesos que originaron el surgimiento de una ocupación, sino que nos permite también su análisis de forma proyectiva, proponiéndonos analizar las diferentes posibilidades que posee una ocupación para desarrollarse, siempre desde un análisis de lo real de la ocupación y no proyecciones o posibilidades que aparecen como irrealizables o imposibles. Sin embargo, estos nuevos elementos, no sólo contribuyen a 
ampliar el espectro temporal de la ocupación, sino también propone descubrirla en tanto poseedora de una esencia, de una característica particular que la hace única frente a otras ocupaciones o frente a otras actividades, así como también nos permite caracterizarla desde sus diferencias con el desempeño de la misma ocupación, pero en contextos distintos - categoría de lo particular - , y al revés, identificar las características de la misma que permiten conocer elementos comunes y no cambiantes de la misma ocupación desarrollada en momentos y lugares diferentes - categoría de lo general-. Es de esta forma, que el análisis a través de las categorías utilizadas permiten ampliar la visión del concepto de ocupación, aportando con nuevos elementos en su análisis, y que consecuentemente nos permite observarla y comprenderla en la práctica como un proceso dinámico y complejo, en el momento que el fin último de las categorías es lograr el análisis de un todo, que en este caso corresponde a la ocupación.

- Tomando en cuenta lo descrito anteriormente, y directamente relacionado con un método de análisis que nos permite, en primera instancia, describir la ocupación desde su dinamismo, es que esta experiencia nos permitió lograr "ejercitar" la capacidad de analizar un fenómeno desde su movimiento. Según el tipo de enseñanza que hemos recibido, la forma de tratar de comprender los fenómenos actualmente está orientada a analizar procesos u objetos estáticos, atemporales, nos provoca dificultad, desde los métodos comunes de análisis, enfrentarnos a un método que tiene como objetivo comprender y generar conocimiento desde lo dinámico de los procesos, ciertamente se suman más elementos, que en un principio significa un aumento de complejidad en el análisis, debido a que corresponde a una forma muy distinta a lo que nos han enseñado y hemos adoptado como "única”. Es por esta razón que significó un desafío y una oportunidad de romper viejas formas, de flexibilizar nuestros mecanismos de pensamiento, de ampliar los razonamientos y de descubrir elementos a considerar que en la cotidianeidad no solemos identificar de forma simple.

- Finalmente otra ventaja es que nos permite analizar desde la práctica a la ocupación, y no partiendo desde ideas preconcebidas o de las voluntades de quien intenta realizar su comprensión, ya que constantemente se dirige a identificar elementos que ciertamente están centrados en el proceso ocupacional, no dando espacio para libres ideaciones del fenómeno.

\section{Desventajas}

La forma de presentación de resultados, basada en la descripción de cada una de las categorías que engloba un análisis dialéctico, se presenta como una propuesta embrionaria, y no exenta de profundos cuestionamientos y aportaciones que vendrán posiblemente a enriquecerla, desde el estudio de la ocupación. Si bien podría significar una propuesta "novedosa” dentro de la escasa aportación teórica en torno al estudio de la ocupación, también ésta presenta potenciales falencias que podrían dificultar su desarrollo, o desincentivar la aproximación por parte de los estudiosos de la ocupación. 
Es de esta forma que se presentan como potenciales desventajas:

- Al contener seis categorías presentadas como "opuestas dentro de una misma unidad categorial”, hace de ésta un elemento de mayor complejidad al momento de describir las ocupaciones, en parte por la necesidad de estudiar en profundidad el desarrollo de la ocupación para luego tener que describirla según cada categoría y su contrario. Esto podría significar una importante inversión de tiempo, más aún si no se está familiarizado con la lógica dialéctica ni sus categorías, al momento de utilizarla en un contexto real.

- Al plantearse desde una etapa inicial de reducción dialéctica, para posteriormente analizar los factores descritos en ella, podría significar, que al no comprender cabalmente el análisis desde este paradigma se podría caer en el error de desarrollar el análisis con los elementos separados, o considerar solo algunos, de manera parcial, o sea, desarrollar la investigación desde el plano de lo abstracto.

- Dentro del mismo plano, cabe destacar, que la enseñanza del método dialéctico, han sido prácticamente censurados y por lo tanto erradicados de la gran mayorías de los sistemas educacionales formales, por lo que plantear investigaciones desde esta perspectiva y conseguir material necesario y de calidad para su estudio y desarrollo, son por decirlo menos dificultosos. Además gran parte de la escasa fuente bibliográfica disponible, ha pasado por la pluma nefasta del dogmatismo soviético, el cual utilizando los conceptos desarrollados por la metodología dialéctica y materialista anteriores, los han logrado sistematizar y cerrar dentro de sus propios límites, desviando y tergiversando de esta manera lo esencial de la misma.

- Dentro de lo mismo es posible que exista poco interés por parte de los estudiosos de la ocupación, en profundizar y desarrollar este incipiente trabajo, ya que además de lo señalado en el punto anterior, no genera ningún resultado numérico ni categorial, ya que no pretende sistematizar, clasificar, ni obtener puntajes. Los Terapeutas Ocupacionales y estudiosos de la Ocupación hasta la fecha, se han vinculado durante mucho tiempo a los instrumentos y enfoques tradicionales, ligados a las perspectivas cuantitativas y cualitativas, por lo que incorporar ésta nueva lógica en sus estudios, puede resultar dificultoso y poco motivante, más aún si no se comprende la lógica subyacente.

\section{CONCLUSIÓN}

Nuestro trabajo sólo pretende ser una pequeña aproximación inicial, en tanto identificación primera de los elementos que interactúan en el fenómeno de la ocupación, y que nos entrega un primer acercamiento a su problemática, por lo tanto comprendemos que este intento corresponde al primer paso de un proceso más extenso y complejo que apunta, en el futuro, hacia una nueva comprensión conceptual del fenómeno ocupacional no explorada hasta el momento.

Si bien, el esclarecimiento epistemológico de este proceso nos dirige hacia una conceptualización del "para qué" de la aplicación de este método en el estudio y desarrollo científico de la ocupación, también nos dirige hacia el esclarecimiento del "para quién” está 
orientada esta propuesta, tarea que no nos corresponde en esta ocasión clarificar, sino más bien nos permite abrir el desafío a aquellos que respondan a la invitación de desarrollar, desafiar, tensionar, dialogar, criticar y aportar desde un método que se nos presenta en movimiento, a relacionarlo con un fenómeno tan complejo como la ocupación, y transitar dialécticamente, desde este estudio exploratorio inicial, que parte desde la práctica de la ocupación como fuente de conocimiento, hacia su problematización social como fenómeno ocupacional, proceso que pugna con el constante peligro que corre la ocupación como concepto, de convertirse en un objeto inmóvil en nuestras cabezas, y desde ahí , hacia una comprensión fosilizada del fenómeno, provocándonos choques o incoherencias cuando nos enfrentamos con ésta en lo real de nuestra cotidianeidad, generando posibles "pérdidas de sentido o propósito" en cuanto al objetivo central del desempeño de nuestra disciplina, y que según la propuesta de este estudio, se dirige hacia un objetivo trasformador y democratizador de las ocupaciones tal cual se manifiestan hoy en nuestro espectro social.

Tal como lo evidenciamos en las ocupaciones descritas en este trabajo, seleccionadas desde textos de la literatura chilena, éstas no solamente nos refieren pasajes aislados de las actividades que realizaban los personajes en un tiempo y espacio determinado, sino que además nos revelan parte de la identidad de los sujetos que se han desarrollado en esos contextos y cómo estos contextos y esas ocupaciones forman parte de los sujetos que se desenvuelven en la sociedad actual a través de sus ocupaciones.

Mediante la comprensión del movimiento en la sociedad, de las relaciones y las contradicciones podemos comprender, por ejemplo, como las ocupaciones de hoy están relacionadas con las ocupaciones de ayer, como las transformaciones que realizó el minero salitrero de ayer, los que sobrevivían al margen de la ley, han sido la antesala, de lo que vivenciamos actualmente, del desarrollo de esta sociedad, del desarrollo de nuestras ocupaciones.

Finalmente, consideramos necesario en cualquier tipo de proceso de comprensión de fenómenos, la autocrítica de lo que se plantea, entendiendo que la compleja tarea de lograr conocer las múltiples interacciones que nos rodean y de las cuales somos parte, no se "encierran" en un aquí y ahora, no tienen un final determinado, ya que los procesos que buscamos conocer están en constante movimiento y transformación, por lo que la autocrítica nos brinda la oportunidad de superación, de constante avance.

Dentro de esta perspectiva, surge que los autores mantuvimos, en la práctica, la división sujeto-objeto, ya que nos apartamos del fenómeno y desde fuera evaluamos una ocupación como objeto, desconociendo el vernos a nosotros mismos en esa ocupación como sujetos históricos, como parte de una misma historia (unión sujeto-objeto). Este hecho es contradictorio en cuanto a la perspectiva dialéctica materialista y responde a visiones reduccionistas que nos invitan a visualizar los fenómenos como objetos aislados e inmóviles dentro de la realidad, además de posicionarnos como meros observadores de fenómenos y no como transformadores. 
Y como consecuencia de esta autocrítica, surge un nuevo cuestionamiento: ¿Será la ocupación un objeto? Ya que cuando hablamos de "objeto", hablamos de "cosa". Pero nuestro objetivo aquí es caracterizar a la ocupación como una relación social, producto y productora de realidad. Por lo que acoger la premisa de que la ocupación es nuestro objeto, estaríamos cosificando e inmovilizando una relación.

Es de esta forma, que a través de la crítica se producen y reproducen nuevas interrogantes, nuevos fenómenos por comprender y se va enriqueciendo y a la vez complejizando el proceso de interpretar, conocer, desarrollar y transformar, en nuestro caso, desde y hacia la ocupación como praxis.

\section{Agradecimientos}

Agradecemos el valioso y sustancial aporte durante el desarrollo del presente trabajo a la T.O. Laura Rueda, T.O. Inés Correa y al T.O. Alejandro Guajardo por el apoyo, la orientación y las enriquecedoras discusiones, que fueron fundamentales para comenzar este proceso de cuestionamiento y construcción en la Terapia Ocupacional y Ciencia de la Ocupación.

Además brindamos un profundo agradecimiento a nuestro gran amigo y colega, T.O. Diego Cifuentes Lucero, por el gran apoyo y participación en todos los proyectos emprendidos.

\section{REFERENCIAS BIBLIOGRAFICAS}

${ }^{1}$ ASTARITA R. Notas sobre la concepción social, fuerzas productivas y relaciones de producción en Marx. Notas de clases. 2006. Disponible en: www.rolandoastarita.com (consultado 27 de septiembre de 2008)

${ }^{2}$ SPIRKIN Y YAKOT. Fundamentos del materialismo dialéctico e histórico. Santiago de Chile: Editorial Austral, 1972; 19-20

${ }^{3}$ TCHAGUIN B.A. Engels y el materialismo histórico, El problema de los factores objetivos y subjetivos en la historia. Buenos Aires. Argentina: Biblioteca del hombre contemporáneo, 1976; 2: 59

${ }^{4}$ PICHÓN RIVIÈRE E, PAMPLONA DE QUIROGA A. Psicología de la Vida Cotidiana. Buenos Aires. Argentina: Editorial Galerna.1970: 10.

${ }^{5}$ SPIRKIN Y YAKOT. Fundamentos del materialismo dialéctico e histórico. Santiago de Chile: Editorial Austral, 1972; 19-20 